\title{
Discours
}

Revue de linguistique, psycholinguistique et

informatique. A journal of linguistics, psycholinguistics and computational linguistics

$9 \mid 2011$

Varia

\section{Entre statut phrastique et statut textuel : l'exemple des énoncés situationnels}

\section{Christiane Marque-Pucheu}

\section{OpenEdition}

Journals

Édition électronique

URL : http://journals.openedition.org/discours/8553

DOI : $10.4000 /$ discours. 8553

ISSN : 1963-1723

Éditeur :

Laboratoire LATTICE, Presses universitaires de Caen

\section{Référence électronique}

Christiane Marque-Pucheu, « Entre statut phrastique et statut textuel : l'exemple des énoncés situationnels », Discours [En ligne], 9 | 2011, mis en ligne le 20 décembre 2011, consulté le 10 décembre 2020. URL : http://journals.openedition.org/discours/8553 ; DOI : https://doi.org/10.4000/ discours.8553

\section{(c) $\oplus \Theta \Theta$}

Discours est mis à disposition selon les termes de la licence Creative Commons Attribution - Pas d'Utilisation Commerciale - Pas de Modification 4.0 International. 

Revue de linguistique, psycholinguistique et informatique

\section{Entre statut phrastique et statut textuel: l'exemple des énoncés situationnels}

Christiane Marque-Pucheu

Christiane Marque-Pucheu, «Entre statut phrastique et statut textuel : I'exemple des énoncés situationnels», Discours [En ligne], 9 | 2011, mis en ligne le 20 décembre 2011.

URL: http://discours.revues.org/8553. Consulté le 20 décembre 2011.

Titre du numéro: Varia

Coordination: Anne Le Draoulec et Josette Rebeyrolle 



\section{Entre statut phrastique et statut textuel : l'exemple des énoncés situationnels}

Christiane Marque-Pucheu

Parmi les énoncés liés à une situation, caractérisés par un figement énonciatif, certains présentent un statut phrastique, d'autres un statut textuel. Les premiers peuvent, par exemple, introduire une modalité (Tu parles (que non)!). Les seconds servent notamment de cadre de discours: l'énoncé Qu'est-ce qui me/nous dit que P? peut ainsi reprendre une information précédente en permettant de développer une argumentation qui va couvrir plusieurs phrases. Délimiter la frontière entre statut phrastique et statut textuel n'est cependant pas toujours trivial. Qu'ils soient dotés d'un statut phrastique ou textuel, ces énoncés ont un fort pouvoir argumentatif. Visant des stratégies différentes, tantôt ils soulignent l'homogénéité argumentative (Je ne te le fais pas dire), tantôt ils introduisent une inversion argumentative (Je ne voudrais pas dire... mais) ou encore, mettant en jeu l'infratexte, ils jouent sur l'implicite: ainsi, dans Je me comprends, le lecteur restitue Tu ne me comprends pas.

Mots clés: argumentation, cadre de discours, figement énonciatif, implicite, infratexte, phrase, texte

Among statements related to a situation and subject to a fixed enunciation, some can be mainly considered as (isolated) sentences, while others are mainly fragments of discourse. The first category can, for example, introduce a modality (Tu parles (que non)!), while the second one has a discourse-framing function: Qu'est-ce qui me/nous dit que P? may take up previous information, enabling an argument to be developed over several sentences. Defining the boundary between sentential and textual status is no easy matter. Both types of statement have a strong argumentative power, and can target different strategies: they sometimes highlight argumentative homogeneity (Je ne te le fais pas dire), while sometimes introducing counter-arguments (Je ne voudrais pas dire... mais); they may also play with implicit messages (infra-text): for example, in Je me comprends, what the reader understands is Tu ne me comprends pas.

Keywords: argumentation, framework of discourse, fixed enunciation, implicit, infra-text, sentence, text

La continuité textuelle, inhérente au texte, suppose que deux conditions soient remplies: l'énoncé - la suite d'énoncés - doit être grammatical et interprétable dans le contexte linguistique (composante locutoire) et dans le contexte énonciatif (composante illocutoire).

Dans le contexte linguistique, cette continuité est assurée en premier lieu grâce à diverses opérations de liage sémantique (Adam, 2005: 85-103), mises en œuvre par le biais de l'anaphore qui reprend l'information et de l'isotopie qui correspond à une redondance sémique. La continuité textuelle est réalisée en second lieu par la progression qu'introduit une information nouvelle (Combettes, 1983) et aussi par l'instauration de cadres de discours (Charolles, 1997) qui «coiffent» des propositions sans introduire de hiérarchisation entre elles. En d'autres termes, un texte doit être structuré. 
Dans le contexte énonciatif, la continuité textuelle est assurée lorsque le lecteur perçoit chez l'énonciateur une intentionnalité globale à laquelle ce dernier subordonne les actes de langage. On a coutume alors de parler de «stratégie argumentative» et celle-ci s'exerce de deux manières.

Une première manière consiste en une stratégie argumentative "par contiguïté» ${ }^{1}$. Les connecteurs peuvent jouer ce rôle de liaison entre propositions grâce à des mots, des groupes de mots, mais aussi - moins souvent citées - des phrases qui sont alors fonctionnellement équivalentes à des mots. La stratégie "par contiguïté» peut être «à orientation homogène», c'est-à-dire que les composantes énonciatives du texte visent une conclusion identique, ou «à réorientation argumentative», c'est-à-dire que deux orientations argumentatives au moins divergent. Selon le cas, les connecteurs sont argumentatifs (donc) ou «contre-argumentatifs» (mais).

Une deuxième manière, plus complexe, consiste en une stratégie argumentative dite «à distance», car l'intention ne se déduit pas directement du contexte immédiat. Elle se déduit d'une autre unité textuelle qui est soit matérialisée au sein du même texte (intratextualité) - par exemple au sein d'un même roman - ou à l'extérieur (intertextualité) - par exemple entre un texte et son pastiche -, soit non matérialisée (infratextualité), par exemple quand le texte est suggéré ${ }^{2}$. En effet, l'interprétation de l'intention se déduit d'une composante implicite, mettant en jeu des mécanismes inférentiels et des implications sous-jacentes. Or, c'est le propre de nombreux énoncés situationnels, c'est-à-dire des énoncés liés à une situation récurrente qui les déclenche automatiquement, qu'ils commentent et dans laquelle ils se sont figés, de «vouloir dire» plus que de dire (Martins-Baltar, 2003; Fónagy, 2005; Marque-Pucheu, 2007). Ces énoncés, qui font appel à l'implicite et ont en quelque sorte un «sens codé (secret)» (Fónagy, 2005: II4) ${ }^{3}$, peuvent donc relever de l'infratextualité.

Si la structuration du texte a été abondamment illustrée, notamment au sein de textes littéraires (Neveu, 1995), et si des mots privilégiés comme donc ou mais ont donné lieu à de nombreuses études pragmatiques (Ducrot, 1980; Rossari, 2000) ${ }^{4}$, il est plus rare de voir mentionner des séquences plus longues ${ }^{5}$. Or, quel que soit le cas - stratégie "par contiguïté» ou «à distance»-, notre hypothèse est que les énoncés situationnels, en étant inscrits dans la situation d'énonciation et en mettant en jeu une interprétation énonciative et pragmatique (notamment argumentative),

1. Nous reprenons la classification et la terminologie utilisées par Soutet (2005:332-335).

2. Soutet (2005: 335$)$ parle de texte «effectif» ou non.

3. Citons un exemple - non organisateur de texte - emprunté à Kerbrat-Orecchioni (2002). Dans un café, le serveur interrogera le client en demandant Vous prenez quelque chose?, ce qui correspond en réalité non pas à une interrogation totale mais à l'interrogation partielle «Que prenez-vous?».

4. Des numéros entiers de revues s'intéressent aux phénomènes de connexion (Langue française, $\mathrm{n}^{\circ} 77,1988$; Syntaxe et sémantique, $\left.\mathrm{n}^{\circ} \mathrm{I}, 2000\right)$.

5. Dostie (200I) fait exception. 
contribuent aussi bien à l'organisation du texte qu'à l'enchaînement local des phrases. Même si la frontière entre phrase et texte n'est pas toujours aisée à délimiter, nous montrons en quoi ces énoncés représentent une classe textuelle.

Après avoir rappelé les caractéristiques des énoncés liés à une situation (partie I), nous illustrerons notre hypothèse dans le domaine de la stratégie argumentative "par contiguïté» (partie 2), avec deux grands axes: énoncés «à orientation homogène» et énoncés «à réorientation argumentative». Dans le deuxième type de stratégie argumentative - «à distance»-, nous laissons de côté intratextualité et intertextualité, qui n'ont aucune pertinence par rapport aux énoncés situationnels, pour nous limiter à l'infratextualité (partie 3), et donc au texte non effectif que peut impliquer un énoncé situationnel.

\section{Les énoncés situationnels}

\subsection{Critères de reconnaissance}

8 Rappelons, à la suite de Fónagy (2005: II4-II8), que les énoncés situationnels sont des expressions convenues inscrites dans la situation d'énonciation (voir ci-dessus), caractérisées à des degrés divers ${ }^{6}$ par quatre types de traits.

\subsubsection{Traits morphosyntaxiques et lexicaux}

Les variations morphosyntaxiques ou lexicales qui peuvent affecter les énoncés situationnels sont limitées: Qu'est-ce qu'on parie? mais non * Qu'est-ce qu'on pariait?; Vous m'en direz des nouvelles mais non *Vous m'en donnerez des nouvelles. Les ellipses sont fréquentes, concernant le complément (Vous voulez que je vous dise (ce que je pense/ma pensée)?), la principale (Si tu le prends comme ça (je vais renoncer)) ou la subordonnée (C'est dire (s'il faisait beau)). Leur syntaxe peut être marquée (De quoi je me mêle??; Toccupe!; Y’a des fois, franchement!).

\subsubsection{Traits sémantico-référentiels}

Leur pouvoir référentiel est généralement affecté dans la mesure où ces expressions sont convenues (Vous m'en direz des nouvelles ( $E /{ }^{*}$ fraîches)), et souvent leur interprétation met en jeu une atténuation du sens et/ou un sens décalé: N'bésitez pas est proche de l'invitation, Faut pas chercher à comprendre note une «réaction [...] devant l'attitude d'un tiers que l'on ne comprend pas ou que l'on n'approuve pas» (Bidaud, 2002, $\mathrm{n}^{\circ}$ 157) et Vous n'auriez pas dî fait office de remerciement.

6. De nombreux linguistes s'accordent sur le caractère graduel du figement (G. Gross, I996; Marque-Pucheu, 1995; Svensson, 2004; Lamiroy et Klein, 2005), contrairement à Dubois et Dubois-Charlier (2004) qui en font l'économie dans leur dictionnaire électronique des locutions.

7. Ici, l'utilisation du pronom je fait une entorse à l'emploi habituel: il «ne désigne pas le locuteur, mais l'allocutaire» (Anscombre, I990: 59). 


\subsubsection{Traits prosodiques}

Des indices prosodiques interviennent dans leur caractérisation. Ainsi, l'intonation de C'est trop facile diffère selon que l'expression est liée (exemple [2]) ou non (exemple $[\mathrm{I}]$ ) à une situation récurrente, en l'occurrence une situation d'excuse:

[I] Tu as déjà fini l'exercice?

C'est trop facile.

[2] Je suis en retard, mon réveil n’a pas sonné.

C'est trop facile!

Dans [I], la réponse, qui constitue une «vraie» réponse, peut être exclamative ou non, dans [2], la réponse, qui correspond à C'est trop facile comme argument/ excuse, est nécessairement exclamative.

\subsubsection{Traits énonciatifs}

Enfin, et c'est sans doute un point déterminant dans la distinction avec les expressions (semi-)figées non liées à une situation, le critère énonciatif intervient doublement. D’une part, un énoncé lié à une situation est associé à une situation stéréotypée (C’est pas un peu fini! marque l'agacement) (voir critère 2) ; son acceptabilité grammaticale est susceptible d'être liée à l'énonciation (Quand même!; Vous voulez que je vous dise?). Le critère énonciatif se manifeste, d'autre part, dans la non-compositionnalité stylistique: l'expression Vous m'en direz des nouvelles appartient à la langue familière quand elle est énoncée, alors que ses composants sont d'un style neutre ${ }^{8}$.

Tout en étant autonomes, puisque leur simple profération suffit à les rendre acceptables (C'est pas pour dire, mais...; Maintenant que j'y pense), les énoncés situtionnels s'inscrivent dans un contexte qui les justifie ou les éclaire: phrastique (Marque-Pucheu, 2007, 2009), mais aussi textuel, notamment par leur fonction de liage. Formellement, ce sont des phrases (même lorsqu'elles sont elliptiques), et fonctionnellement ces phrases ont un rôle organisateur qui va de la liaison locale entre propositions à une structuration plus étendue concernant le texte.

\subsection{Remarques sur le corpus}

Nous avons puisé nos exemples essentiellement dans les dialogues de la conversation courante. La linguistique textuelle en effet ne se limite pas aux textes présentant une qualité esthétique; et par ailleurs, les expressions formulaires (situationnelles) appartiennent souvent à l'oral. À cet égard, la base textuelle FRANTEXT n'offre pas toujours d'occurrences de ces expressions qui dépassent pourtant largement le

8. Les grammaires de construction (Fillmore, Kay et O'Connor, 1988) s'avèrent particulièrement pertinentes pour rendre compte des énoncés situationnels. En effet, celles-ci considèrent que syntaxe, sémantique, pragmatique, discours, morphologie, phonologie et prosodie contribuent de manière égale à leur analyse. 
millier ${ }^{9}$, le roman populaire n'y représentant qu'une faible part. Mais les dictionnaires également sont inégaux, voire muets. Le Dicomotus de Martins-Baltar (20II) pourrait constituer un vivier précieux car il évite de confondre figement strict et figement énonciatif en ne retenant que les expressions situationnelles, mais il se contente de donner des synonymes; de plus, il n'offre pas d'exemple en contexte et aucune des expressions que nous avons choisies n'y figure.

Des études linguistiques traitent aussi de cette question. Bidaud (2002), qui, comme Martins-Baltar (20II), s'en tient au figement situationnel, donne près d'un millier d'exemples en contexte (sous forme de dialogues) et donc en discours. M. Gross (1986) classe les expressions selon leurs propriétés en distinguant notamment des adverbiaux à contenu phrastique, mais le contexte correspond à un contexte phrastique et n'envisage donc pas de suite plus longue, qu'il s'agisse d'un contexte qui précède ou qui suit l'expression concernée. La démarche est identique chez Dubois et Dubois-Charlier (2004) qui cherchent à décrire les patrons («schèmes») syntaxiques dans lesquels s'insèrent les locutions, et parmi elles, sans qu'elles soient nommées comme telles, les expressions situationnelles (Marque-Pucheu, 20Io). Il a donc été nécessaire de forger parfois de toutes pièces un certain nombre de contextes ${ }^{10}$. La tâche n'est pas facile: en effet, si le linguiste est rompu aux techniques de fabrication de phrases, il est plus difficile d'en envisager une suite plausible.

\section{Stratégie argumentative "par contiguïté »}

Nous avons indiqué en introduction que la cohésion du texte était obtenue, dans le contexte énonciatif, quand on percevait chez l'énonciateur une intentionnalité globale et que cette dernière pouvait passer par deux types possibles de stratégie de sa part. Examinons-les en vérifiant comment les énoncés situationnels assurent cette cohésion.

\subsection{Stratégie «à orientation homogène »}

Dans ce premier cas, les composantes énonciatives du texte considéré visent une conclusion identique. Environ dix pour cent de notre corpus servent cette stratégie, s'appliquant, selon le cas, à un segment gauche ou droit du discours.

\subsubsection{Portée sur un segment antérieur ou postérieur \\ 2.1.1.1. Qu'est-ce qu'on parie!: instauration d'un cadre de discours}

Dans les expressions Qu'est-ce qu'on parie!, Qu'est-ce que tu paries!, On/Je parie que P!, parier signifie «affirmer avec vigueur» " et, plus littéralement, "prendre un

9. Bidaud (2002) à elle seule en comptabilise plus de 1300 sans pour autant incorporer le Dicomotus de Martins-Baltar (20II)

10. Voir, notamment, 2.I.I.2, 2.2.I.I, 2.2.2.

11. Le nouveau Petit Robert. Les données électroniques fournies par l'école de M. Gross et de ses successeurs sur http://infolingu.univ-mlv.fr/ donnent parier sous la forme être prêt à parier. 
pari», "proposer un pari à quelqu'un», lorsqu'on estime pouvoir gagner à coup sûr. Mais Je parie se distingue de cette paraphrase puisque J'affirme n'accepte pas le type de phrase interrogatif:

Je te parie que $P$ ?

${ }^{*}$ Je t’affirme que $\mathrm{P}$ ?

Les expressions mettant en jeu parier ne sont pas pour autant des interrogations classiques. Dans l'exemple:

Mais si, papa, tu verras, qu'est-ce que tu paries qu'un jour on l'aura? Mais ça ne se fera pas tout seul, je le sais bien. Il y aura des difficultés...

(N. Sarraute, Le planétarium, 1959)

aucune réponse du type Je parie une bouteille de champagne n'est attendue. L'interrogation partielle en Qu'est-ce que est donc purement formelle. Elle est d'ailleurs équivalente à une interrogation totale puisqu'il est possible de faire commuter $T u$ paries? et Qu'est-ce que tu paries? ${ }^{\text {?2. }}$. Ce sont des interrogations rhétoriques. La même analyse devrait pouvoir s'appliquer quand le pronom est $j e$ :

- Voyons... Voyons... disait Manouche. Fais attention, ils vont t'entendre.

- Et après? Est-ce que nous ne les entendons pas, nous? Tiens, voilà le type qui tousse. Je te parie qu'il va ronfler!

(M. Genevoix, Les mains vides, 1928)

où Je te parie commuterait avec Qu'est-ce que je te parie?! Toutefois, nous n'avons trouvé aucune occurrence de Qu'est-ce que je parie?! alors qu'on accepte Qu'est-ce qu'on parie?!

On dispose d'un argument en faveur du statut phrastique. Sur le plan fonctionnel, Je te parie est un adverbial ${ }^{13}$ comme pour sûr, spécifiant l'attitude du locuteur vis-à-vis de la proposition qu'il peut précéder ou suivre (Il va ronfler, je te parie et Je te parie (qu')il va ronfler?). Et, de même que Tu penses (voir ci-dessous, 2.I.I.2), l'expression peut être attribuée aussi bien au locuteur I:

- Je te parie qu'Ast ne viendra pas dimanche, dit Agnès.

- Pourquoi pas? dit Noémi.

(R. Queneau, Les enfants du limon, 1938)

qu'au locuteur 2, comme dans la citation de Genevoix ci-dessus.

Cette communauté de fonctionnement avec un adverbial suggère une modalité. Or, la modalité se limite en principe au contenu propositionnel. De fait, le pari

12. Toutefois, l'interprétation change légèrement: Qu'est-ce que tu paries? signifie Tu peux parier (beaucoup, tout ce que tu veux, car je tiens le pari-je suis sûr de gagner).

13. Même s'il garde sa fonction de verbe. En incise, comme tout modalisateur épistémique, il perd son complémenteur. 
correspond à un calcul (probabiliste) sur le contenu propositionnel. Un exemple comportant un anaphorique comme je l'aurais parié dans

- Je suis ingénieur chimiste.

- Je l'aurais parié, dit Lucas.

(R. Queneau, Loin de Rueil, 1944)

va dans ce sens.

On pourrait en conclure que la portée de Je te parie ne s'étend pas au-delà de la proposition.

Mais parier suppose un allocutaire - voire plus, un partenaire dans le pari -, ce qui n'est pas le cas avec affirmer:

On parie que $\mathrm{P}$ ?

* On affirme que $\mathrm{P}$ ?

On inclut je et $t u$ avec parier, alors que on correspond uniquement à un tiers (les gens) avec affirmer. Le fait qu'un allocutaire soit impliqué ouvre la possibilité d'une suite/ réaction ${ }^{14}$ et va dans le sens d'un statut textuel de l'expression. Je/On parie que $P$ peut donc constituer un introducteur d'univers de discours dans lequel un locuteur prend en charge et fait prendre en charge à un allocutaire une/des propositions sous la portée du pari, en cherchant à imposer à ce dernier une orientation argumentative. Dans ce sens, on peut dire que Je te parie et On parie ont une dimension textuelle.

\subsubsection{Tu penses!: annonce d'une séquence explicative}

Le fonctionnement de Tu penses! est identique à celui de Je (te) parie (que P)! dans la mesure où l'expression peut s'observer en contexte droit:

Ce ne sont pas les idiots ou les simples canailles qui me donnent beaucoup de souci, tu penses! à quoi serviraient les saints?

(G. Bernanos, Journal d'un curé de campagne, 1936)

ou en contexte gauche. Dans ce dernier cas, l'expression peut introduire une interrogative indirecte:

Tu penses (s'il a accepté/combien il était heureux)!

Cette possibilité n'est pas systématique quand Tu penses! est en contexte droit:

?S'il a accepté, tu penses!

${ }^{*}$ Combien il était heureux, tu penses!

14. S'il n'y a pas lieu de dédoubler On parie que $P$ et $Q u$ 'est-ce qu'on parie que $P$ !, il se peut que Qu'est-ce qu'on parie que $P$ ! soit plus fréquent. Moreau (I988) a attiré l'attention sur le fait que dans les pratiques langagières, un mot peut être employé préférentiellement dans telle ou telle position syntaxique ou telle ou telle construction. 
Synonyme de en effet, évidemment, l'expression introduit une «forte affirmation: comme tu peux le penser» ${ }^{15}$. On pourrait conclure à une portée phrastique, l'expression s'analysant comme:

Tu penses que oui.

Mais le locuteur justifie généralement ensuite par un car (exemple [3]) ou un c'est bien pourquoi (exemple [4]):

[3] Je t'ai suivie, parce que je n'osais te serrer la main devant le monsieur qui était là. Et j’avais une envie d'être seule avec toi, tu penses! Car, ici, je ne vois plus personne de notre ancien monde.

(É. Zola, Madeleine Férat, 1868)

[4] Mais ils ont bien tenu à préciser d'avance qu'ils me paieraient, tout comme les autres ouvrières. Tu penses! C'est bien pourquoi j'y suis allée.

(M. Genevoix, Les mains vides, 1928)

Si la justification par car peut être faite sans Tu penses!, l'expression renforce cette fonction explicative et le rôle de connexion textuelle apparait clairement. Tout en reprenant un contexte antérieur comme dans:

- Il a accepté.

- Tu penses (s'il a accepté)!

l'expression peut donc aussi donner lieu ensuite à une «séquence explicative» (Adam, 2005: $162-163)^{16}$.

\subsubsection{Portée sur un segment strictement antérieur}

Contrairement à Je te parie (que P) qui peut être attribuée au locuteur I ou 2 (voir 2.I.I.I), l'expression Je ne te le fais pas dire! est nécessairement proférée par le locuteur 2:

Locuteur 1 - Ce que je peux être bête! dit-il en choisissant la seconde voie.

Locuteur 2 - On te le fait pas dire, dit Chantal qui l'avait rejoint avec les deux autres femmes.

Locuteur 3 - Et ce taxi? demanda Julia. Faut-il que je m'en occupe?

(R. Queneau, Le dimanche de la vie, 1951)

En effet, ici la continuité textuelle est assurée par le pronom le qui reprend l'information de la phrase précédente. L'expression, qui exprime une approbation

15. Dictionnaire encyclopédique Quillet.

16. Toutefois, Tu penses!, qui n'a pas nécessairement la seule valeur de «forte affirmation» observable dans [3] et [4] et paraphrasable par Comme tu peux le penser, a aussi une acception qui se rapproche de la valeur la plus courante de $T u$ parles, exprimant alors un refus (Bidaud, 2002, $\mathrm{n}^{\circ} 643$ ). 
emphatique et traduit une stratégie «à orientation homogène», a un fort pouvoir conclusif:

[5] - Enfant de putain!

Il s'est esclaffé:

- Je ne te le fais pas dire.

Gina n'a rien dit, paraissant ailleurs.

(L. Malet, Le soleil n'est pas pour nous, 1949)

[6] - Elle est malheureuse. Elle a décidé qu'aujourd'hui c'était le printemps.

- Mais alors elle va de plus en plus mal?

- Je ne te le fais pas dire.

Il se rassied épuisé.

(C. Paysan, Les feux de la Chandeleur, 1966)

Tout contexte droit ne peut qualler dans le sens de cette approbation, comme l'indiquent les séquences Gina n'a rien dit en [5] ou $\mathrm{Il}$ se rassied en [6].

Dans ces quelques exemples, les énoncés situationnels contribuent à la structuration textuelle en traduisant une stratégie homogène. Cette stratégie était perceptible dans le contexte phrastique immédiat, mais nous allons voir qu'elle peut s'étendre sur plusieurs phrases, justifiant la notion de cadre de discours introduite en 2.I.I.I. Nous l'illustrons sur des énoncés qui introduisent cette fois une contre-argumentation.

\subsection{Stratégie «à réorientation argumentative »}

Comme en 2.I, nous pouvons observer des interrogations rhétoriques. Elles revêtent un caractère polémique.

\subsubsection{Inversion argumentative}

2.2.1.1. Polémique avec l'interlocuteur

L'exemple de Vous voulez que je vous dise/Tu veux que je te dise? est intéressant à double titre ${ }^{17}$. Les séquences [7] et [8] font apparaître des relations question-réponse particulières.

En effet, la cohésion textuelle est normalement respectée quand une assertion fait suite à une question. Or, dans [7], la question reste sans réponse. Et dans [8], le couple question-réponse est également anomal, une question faisant suite à une question:

17. La source de cette expression vient sans doute de dire sa pensée:

- En as-tu le pressentiment, Joseph?

- Oui, je l'ai. Mais si tu veux que je te dise toute ma pensée, je crois que cette lettre sera toute différente de celle que tu attends.

(G. Sand, Consuelo, 1843, t. II) 
[7] Locuteur I - Vous voulez que je vous dise?

Locuteur $2-\ldots$

[8] Locuteur I - Qu'est-ce que vous pensez de la dernière proposition de la (majorité/opposition)?

Locuteur 2 - Vous voulez que je vous dise?

Enfin, le locuteur peut répondre à «sa question» sans y avoir été convié, comme dans [9]:

[9] - Et quand je pense, répliquait Brian, que toi, le filleul d'un McNeill, tu ferais tirer sur les Anglais et sur les Écossais!

- Tu veux que je te dise? Tes Écossais n'ont qu'une chose à faire: s'allier aux Irlandais pour lutter contre les Anglais.

(J. d'Ormesson, Le vent du soir, 1985)

Vous voulez que je vous dise? introduit donc une organisation atypique du texte. Mais en l'utilisant, le locuteur I (dans [7]) ou 2 (dans [8] ou [9]) réoriente plus ou moins, selon le cas, l'argumentation de l'interlocuteur, l'expression allant plutôt dans le sens d'une reformulation précisant la pensée du locuteur. Des exemples plus polémiques se trouvent dans Bernet et Rézeau (1995: 88):

- Faites chier. Le tueur du squat, vous voulez que je vous dise?... C'est tout à fait votre portrait.

L'intention polémique est encore plus nette dans l'expression que nous allons étudier.

2.2.1.2. Polémique avec l'interlocuteur ou un tiers

Qu'est-ce qui dit que P? peut introduire une polémique avec l'interlocuteur, sans que l'allocutaire soit mentionné explicitement par te ou vous:

Locuteur 1 - Qu'est-ce qui dit que P?

Locuteur 2 - (Silence possible ou réponse attendue: «Rien».)

L'expression oriente vers la négation: Rien ne dit que $P^{18}$. La polémique est plus réelle si l'on implique l'allocutaire en s'adressant explicitement à lui (te):

Qu'est-ce qui te dit que P ?

Une différence avec Tu veux que je te dise? (2.2.I.I) vient du fait que, dans ce premier cas, la polémique intervient nécessairement entre deux locuteurs en présence. Or, Qu'est-ce qui nous dit que P? peut créer une connivence au sujet d'une polémique avec

18. Le phénomène s'observe souvent avec d'autres types d'interrogation comme Qu'est-ce que je peux y faire? ou Que veux-tu y faire? 
un tiers non présent. Cette connivence apparait d'autant plus nettement que le pronom nous est utilisé (Qu'est-ce qui nous dit?). Dans la polémique qui s'instaure alors avec un tiers, il n'est pas nécessaire que l'allocutaire soit un opposant dans l'argumentation ${ }^{19}$.

Quoi qu'il en soit, Qu'est-ce qui nous dit que P? peut instaurer un cadre de discours. Ainsi, la portée de l'expression s'étend sur un paragraphe entier dans l'interview ci-dessous:

Quand vous entamez votre campagne contre la baisse d'impôts, vous détenez donc déjà des chiffres plus favorables. Pourquoi les avoir ignorés?

Mais qu'est-ce qui nous dit que les cbiffres de septembre étaient plus corrects que ceux de juillet? Ils sont à $100 \%$ estimés et de nouvelles prévisions étaient annoncées. Ce n'est que dans trois ans, quand l'année comptable 2009 sera reconstituée, qu'il sera possible de les vérifier. Et si, alors, ils se révèlent inexacts, peut-être que les 40 millions perdus à cause de la baisse d'impôts nous feront cruellement défaut. Par ailleurs, notre campagne contre la baisse fiscale ne s'est pas articulée autour du déficit, mais autour du manque à gagner de 40 millions qui représentent des prestations. Nous n'avons pas menti : dans notre budget, il n'y a pas de municipalisation des crèches, de projet pour les mamans de jour, d'amélioration de la prise en charge périscolaire...

Salerno: «Qu'est-ce qui nous dit que les chiffres du canton sont corrects».

(Tribune de Genève, 04/11/2009)

Il en va de même ci-après, mais ce n'est plus le jeu des pronoms qui intervient, et la divergence entre les orientations argumentatives est plus forte.

\subsubsection{Conclusion divergeant avec la conclusion implicite}

Contrairement à l'exemple précédent, l'expression Je (ne) voudrais pas dire peut comporter un mais contre-argumentatif. En effet, la conclusion ne correspond pas à la conclusion implicite découlant de Je (ne) voudrais pas dire, qui serait Je ne dis pas $^{20}$. Elle exprime un avis qui vient relativiser une information donnée dans le contexte gauche comme dans:

19. Si nous peut inclure l'allocutaire, qui alors est censé faire bloc avec le locuteur, il peut aussi référer à un nous collectif excluant l'allocutaire, par exemple dans Qui nous dit que vous dites la vérité?

20. L'expression littérale appelle un mais:

Ainsi en Allemagne, loin de demander la liberté politique, elle a accepté, je ne voudrais pas dire la servitude politique, mais l'absence de la liberté.

(F. Guizot, Histoire générale de la civilisation en Europe depuis la cbute de l'Empire romain jusqu'à la Révolution française, 1828)

On remarquera par ailleurs que la forme elliptique s'est fixée au conditionnel (Je ne voudrais pas dire...) et non pas au présent $\left({ }^{*}\right.$ Je ne veux pas dire...) qui nécessite une suite (que $P$ ):

Je ne veux pas dire qu'il ne luy soit arrivé des avantures assez plaisantes depuis son mariage, mais ses amys particuliers en ont esté les seuls tesmoins [...].

(C. Sorel, Le berger extravagant, 1627)

Je ne veux pas dire que tu sois née princesse dans l'acception aristocratique du mot, cela serait une pauvre flatterie à te faire, mon enfant... mais tu es de ce petit nombre d'êtres privilégiés [...].

(E. Sue, Les mystères de Paris, 1843) 
Ses parents sont fiers qu'il ait décroché son bac. Je voudrais pas dire, mais il s'y est repris à deux fois.

L'énoncé indique bien une rupture dans l'argumentation (Je voudrais pas dire, mais je dis quand même) ${ }^{21}$, moindre toutefois qu'avec un simple mais. Remarquons que cette expression n'est pas du même type que les précédentes, car elle n'est pas autonome dans l'exemple donné, $P$ en faisant partie. Néanmoins, on peut l'imaginer en réponse dans un dialogue:

- Ça, c'est un exploit!

- Je voudrais pas dire...

Un petit paradigme avec dire illustre un même cas de figure: on peut pas dire, y'a pas à dire, cela va sans dire, c(e n)'est pas pour dire.

Jusqu'à présent, les exemples cités étaient non ambigus, sauf dans le cas de $T u$ penses! (voir ci-dessus, note I6) ou Tu parles! Or, en l'absence de contexte suffisant, un même énoncé peut prendre deux interprétations opposées.

\subsection{Tu parles!: stratégie «à orientation homogène » ou «à réorientation argumentative »}

Une illustration peut être donnée par Tu parles! En effet, l'expression peut traduire une stratégie «à orientation homogène» ou une stratégie «à réorientation argumentative», signifiant respectivement bien sûr (comme en [Io]) ou pas du tout (en [II]) selon l'intonation:

[ıo] Bon! Bien! Ça va! que je conclus. Et sa mère alors? Elle a dû faire un peu de bruit aussi la mère, quand elle a compris que tu te débinais pour de bon?...

- Tu parles! Même qu'elle répétait toute la journée que j’avais un caractère de cochon [...].

(L.-F. Céline, Voyage au bout de la nuit, 1932)

[II] [...] depuis que la science et la philosophie modernes se donnent des airs de marcher vers l'Inconnu et de l'accueillir à bras ouverts. Tu parles! Elles l'accueillent à coups de bambou.

(J. Romains, Psyché, I929, t. III, Quand le navire...)

Dans le premier cas, $T u$ parles! est intensif et conclusif et correspond à une interprétation positive (Tu parles que oui !):

[roa] Tu parles (qu'/si) elle a fait du bruit! = (Bien sûr qu')elle a fait du bruit.

21. Une autre manière d'introduire une rupture dans l'argumentation consiste à «désasserter». S'agissant de Il pleut. C'est Pron qui le dit, Laurendeau (2009: 6I) parle de désassertion polémique stricte par rapport à Il paraît qu'il pleut (désassertion constative stricte), l'assertion polémique stricte étant Je crois vraiment qu'il pleut, et l'assertion constative stricte Il pleut. 
Dans le second, Tu parles! introduit une contestation, une mise en doute, voire une négation (Tu parles que non!) et peut relancer le dialogue:

[ıг] Tu parles qu'elles l'accueillent à bras ouverts! = (Bien sûr qu')elles ne l'accueillent pas à bras ouverts ${ }^{22}$.

Il est difficile de faire correspondre des propriétés syntaxiques à ces interprétations opposées. Les propriétés de Tu parles (que/si/comme) P! ou Tu parles d'un $N$ ! ne permettent pas de sélectionner une interprétation de préférence à une autre. Mais c'est le propre des phrases exclamatives que de pouvoir acquérir une valeur ironique.

L'origine de ces emplois exclamatifs pourrait être une construction en de comme dans [I2] :

[I2] - Le jour du soleil n'est-il pas assez long pour te plaindre?

- Tu parles de repos! me répondit-elle; il n'y en a plus pour moi sur la terre; mon esprit est dans les ténèbres; les nuages obscurcissent le soleil de ma vie [...].

(M.J. de Crèvecour, Voyage dans la Haute Pensylvanie et dans l'État de New York, I8oI, t. I)

Proféré comme exclamation (voir en [12]), Tu parles de N! acquiert une interprétation ironique. Puis le complément, facilement reconstituable grâce au contexte gauche, est élidé. L'expression Tu parles! devient autonome. Le complément élidé pourrait aussi être phrastique $(Q u e / S i) P^{23}$.

Comme il ressort des différents exemples étudiés, nombre d'énoncés liés sont construits sur des mécanismes inférentiels. Cette caractéristique est encore plus à l'œuvre quand il s'agit de stratégie «à distance».

\section{L'infratexte}

L'expression Je me comprends est figée à la personne I (* Tu te comprends; * Il se comprend, etc. $)^{24}$ et au présent ( ${ }^{*}$ Je me comprenais, ${ }^{*}$ Je me comprendrais). Dans une des premières occurrences, Je me comprends était déductible d'un environnement où elle s'opposait à $T u$ ne me comprends pas:

Tu dois me trouver bête à faire pitié et, si tu ne me comprends pas, je me comprends, hélas, fort bien pour mon malheur.

(G. Flaubert, Correspondance (1840-1845), 1845)

22. C'est nous qui soulignons.

23. L'exemple Parlez-moi de SN, Parlez-m'en pourrait également servir d'illustration.

24. Mais il faudrait noter qu'On se comprend existe aussi avec une valeur un peu différente, exprimant une connivence entre interlocuteurs, vis-à-vis d'un tiers. 
Puis l'expression est devenue «autonome». Elle correspond à un «dédoublement» du discours dans lequel l'énonciateur émet un commentaire sur sa propre parole au moment où il la produit, contribuant ainsi à une meilleure lisibilité textuelle:

[13] Au fond, l'amour n'est pas un sentiment naturel. Enfin, je me comprends, c'est un sentiment nécessaire, on ne peut pas y échapper.

(R. Vrigny, La nuit des Mougins, 1963)

L'unité textuelle complète dans laquelle s'inscrit Je me comprends suppose Tu ne me comprends pas, étant paraphrasable par Je sais ce que je veux dire même si tu ne me comprends pas. En l'absence de Tu ne me comprends pas, un C'est-à-dire (en [I4]) ou un Je veux dire (en [I5]) ou encore un Enfin explicatif (en [I3] ci-dessus) sont mentionnés:

[I4] Le Marchand - Oui, oui, comme la danse, le cheval, le jeu de paume et tant d'autres. Qu'entendez-vous vous-même, père Mondella?

L'Orfèvre - Cela suffit; - je me comprends. - c'est-à-dire que les murailles de tous ces palais-là n'ont jamais mieux prouvé leur solidité.

(A. de Musset, Lorenzaccio, I834, I, 2)

[I5] Bonichot (riant) - Saprée Cadie, va! où que vous voudriez qu'il l'eusse?

Léocadie - Enfin, je me comprends. Je veux dire qu'il est noir de cheveux et pas haut en couleurs.

(G. Chepfer, Saynètes, paysanneries I, 1922)

[16] Au fond, l'amour n'est pas un sentiment naturel. Enfin, je me comprends, c'est un sentiment nécessaire, on ne peut pas y échapper.

(R. Vrigny, La nuit des Mougins, 1963)

La séquence «complémentaire» Tu ne me comprends pas est le plus souvent absente, devenant non effective. Je me comprends suggère donc un infratexte ( $T u$ ne me comprends pas). Bidaud (2002, $\mathrm{n}^{\circ}$ I58) va encore plus loin, donnant l'interprétation «Je sais de quoi je parle mais je n'en dirai pas plus».

D'autres exemples pourraient illustrer l'infratexte: C'est toi qui le dis qui suppose un Ce n'est pas moi qui le dis, car «le locuteur [...] se dissocie de ce qui vient d'être dit» (Bidaud, 2002, $\mathrm{n}^{\circ}$ 253), ou encore Ça s'appelle «revient» qui suppose Ça ne revient pas toujours.

On remarquera que dans la totalité des cas, l'infratexte correspondant est constitué chaque fois par le «négatif» de l'expression considérée.

\section{Conclusion}

Les énoncés étudiés ont bien le statut de phrase puisque, en dépit de leur forme souvent elliptique, parfois agrammaticale, leur seule énonciation les rend acceptables. 
Certains sont porteurs de modalité et, à ce titre, ont une portée plutôt phrastique ( $J e$ ne te le fais pas dire!) que textuelle. Mais d'autres s'inscrivent aussi dans un ensemble plus long, donc de manière plus générale dans un texte, que le contexte soit effectif comme dans Tu parles!, ou non, comme dans Je me comprends qui suppose un texte non réalisé (Tu ne me comprends pas).

Ils contribuent diversement et de manière complexe à la cohérence (énonciative) et à la cohésion (linguistique) du texte. Diversement, car les uns ont une fonction prospective (Qu'est-ce qu'on parie?), les autres une fonction conclusive (Tu penses.). De manière complexe, car la valeur locutoire de ces «micro-actes de discours» (Adam, 2005: 78) est plus ou moins facile à cerner en raison des implicites qui leur sont souvent attachés. Un énoncé positif comme Je me comprends implique un énoncé négatif comme Tu ne me comprends pas.

Bien que le domaine ait été largement défriché sur le plan pragmatique par Martins-Baltar (1994, 2003), la grammaire phrastique et textuelle mise en jeu par ces énoncés reste à décrire.

\section{Bibliographie}

\section{Références}

AdAm, J.-M. 2005. La linguistique textuelle. Introduction à l'analyse textuelle des discours. Paris: Armand Colin.

Anscombre, J.-C. 1990. Thème, espaces discursifs et représentations événementielles. In J.-C. ANSCOMBRE et G. ZACCARIA (eds), Fonctionnalisme et pragmatique. À propos de la notion de thème. Milan: Unicopli : 43-150.

BIDAUD, F. 2002. Structures figées de la conversation. Analyse contrastive français-italien. Berne - Berlin - Bruxelles: Peter Lang.

Charolles, M. 1997. L'encadrement du discours - univers, champs, domaines et espaces. Cabiers de recherche linguistique 6: $\mathrm{I}^{-} 73$.

Cомветтеs, B. 1983. Pour une grammaire textuelle: la progression thématique. Bruxelles: Duculot.

DostiE, G. 200I. La gradation du sens et ses traces morphologiques et syntaxiques. Considérations sur la (poly)pragmaticalisation. Travaux linguistiques $d u$ Cerlico I4: 6I-9I.

Ducrot, O. (ed.) 1980. Les mots du discours. Paris: Éditions de Minuit.

Fillmore, C., Kay, P. et O'Connor, P. 1988. Regularity and Idiomaticity in Grammatical Constructions: The Case of Let Alone. Language 64 (3): 50I-538.

Fónagy, I. 2005. Dynamique et changement. Louvain - Paris: Peeters.

GRoss, G. 1996. Les expressions figées en français. Noms composés et autres locutions. Gap - Paris: Ophrys.

Gross, M. 1986. Grammaire transformationnelle du français. Paris: ASSTRIL. Vol. 3 : Syntaxe de l'adverbe. 
Hansen, M.-B.M. 2008. Particles at the Semantics/Pragmatics Interface: Synchronic and Diachronic Issues. A Study with Special Reference to the French Phasal Adverbs. Current research in the semantics/pragmatics interface 19. Amsterdam - New York - Paris: Elsevier.

Kerbrat-Orecchioni, C. 2002. Politesse en deçà des Pyrénées, impolitesse au-delà: retour sur la question de l'universalité de la (théorie de la) politesse. Marges linguistiques. Accessible en ligne à l'adresse suivante : http://icar.univ-lyonz.fr/Equipes/documents/ MargesKerbrat.pdf (consulté le I4/ı2/20II).

Klein, J.R. et Lamiroy, B. 20II. Routines conversationnelles et figement. In J.-C. AnsCOMBRE et S. MejRI (eds), Le figement linguistique : la parole entravée. Paris: Champion: 195-209.

Lamiroy, B. et Klein, J.R. 2005. Le problème central du figement est le semi-figement. Linx 53: 135-154. Accessible en ligne à l'adresse suivante: http://linx.revues.org/27I (consulté le I4/I2/2OII).

LAURENDEAU, P. 2009. Préassertion, réassertion, désassertion: construction et déconstruction de l'opération de prise en charge. Langue française $162: 55-70$.

Marque-Pucheu, C. 1995. Structures prépositionnelles semi-figées Prép $C_{I}$ de $N_{2}$ : verbes supports associés et structure interne. Thèse de doctorat non publiée. Département des sciences du langage, Université Paris VIII.

Marque-Pucheu, C. 2007. Les énoncés liés à une situation: mode de fonctionnement et mode d'accès en langue 2. Hieronymus I : 25-48. Accessible en ligne à l'adresse suivante: http://www.unizd.hr/portals/43/broj_I_2007/Christiane_Marque_Pucheu_ Les_enonces_lies_a_une_situation.pdf (consulté le I4/I2/20II).

Marque-Pucheu, C. 2009. Mots-phrases, expressions situationnelles: figement énonciatif et changement de portée. Arena romanistica 4: 224-233.

Marque-Pucheu, C. 20io. Exhaustivité et représentativité des expressions comportant dire dans Locutions en français de J. Dubois et F. Dubois-Charlier. Langages 179/I80: 259-280.

Martins-Baltar, M. 1994. Analyse motivationnelle du discours. Paris: CREDIF - Hatier-Didier.

Martins-Baltar, M. 2003. Implicite et culture des énoncés. In M.T. Lino et J. Pruvost (eds), Mots et lexiculture. Paris: Champion: $155^{-222 .}$

Moreau, M.-L. 1988. Un mot, des fonctions, des contextes. Linguisticae Investigationes 12 (I) : I29-I5O.

Neveu, F. 1995. Progressions et ruptures thématiques. Aspects de la technique descriptive dans La Condition bumaine. L'information grammaticale 67: 38-4I.

Ponchon, T. 2005. L'adverbe bien en français médiéval: des signifiés d'effet au signifié de puissance. In O. Soutet (ed.), La polysémie. Paris: Presses universitaires Paris-Sorbonne: $245^{-264}$.

Rossari, C. 2000. Connecteurs et relations de discours: des liens entre cognition et signification. Nancy: Presses universitaires de Nancy.

Soutet, O. 2005. Linguistique. Paris: PUF.

Svensson, M.H. 2004. Critères de figement. L'identification des expressions figées en français contemporain. Umeå: Umeå Universitet. 


\section{Corpus, dictionnaires, ressources électroniques}

ATILF. Base textuelle FRANTEXT. Accessible en ligne à l'adresse suivante: http://www. frantext.fr/.

Bernet, C. et RéZEAU, P. (dir.) 1995. Richesses lexicales du français contemporain. Paris: Klincksieck.

Dubois, J. et Dubois-Charlier, F. 2004. Locutions en français. Aix-en-Provence: chez les auteurs.

Martins-Baltar, M. 20II. Dicomotus. Dictionnaire des expressions de motif usuelles et autres expressions usuelles représentant des conditions d'action. Essai de pragmatographie hypertextuelle explicative. Accessible en ligne à l'adresse suivante: http://michel.martinsbaltar.perso. sfr.fr/ds/dicomotus.html (consulté le is/or/20II).

MorTIER, R. (dir.) 1953. Dictionnaire encyclopédique Quillet. Paris: A. Quillet.

Moteur de recherche Google.

Rey, A. et Rey-Debove, J. (dir.) 1994. Le nouveau Petit Robert. Paris: Dictionnaires Le Robert.

Site de l'équipe d'Informatique linguistique: http://infolingu.univ-mlv.fr/. 